Bol. Acad. peru. leng. 55. 2013 (217-237)

\title{
LÉXICO DEL PROCESO DE FABRICACIÓN DE VINOS PERUANOS
}

\section{LEXIQUE DU PROCESSUS DE FABRICATION DE VINS PÉRUVIENS}

\section{GLOSSARY OF MANUFACTURING PROCESS OF PERUVIAN WINES}

\author{
Zamia Ivón Castillo Farroñay \\ Universidad Nacional Mayor de San Marcos
}

\section{Resumen:}

En esta ponencia se presentará el recojo de una parte del léxico relacionado con el proceso de fabricación de los vinos peruanos con el objetivo de lograr un conocimiento básico sobre este tema. Para la recolección de datos, se consideró necesario elaborar un cuestionario cuya aplicación se llevó a cabo en algunas bodegas ubicadas en la ciudad de Lima. Luego de haber recolectado el corpus necesario se procedió a sistematizarlo siguiendo los diferentes criterios estipulados por la Real Academia Española (RAE) en la última edición del Diccionario de la lengua española.

\section{Résumé:}

Nous présentons dans cet article le recueil d'une partie du lexique se rapportant au processus de fabrication des vins péruviens, dans le but d'obtenir une connaissance de base sur ce sujet. Pour le recueil de données, nous avons considéré nécessaire de construire un questionnaire qui a été appliqué chez quelques vinificateurs de Lima. Après avoir collecté le corpus nécessaire, celui-ci a été systématisé en suivant les différents 
critères stipulés par l'Académie Espagnole (Real Academia EspañolaRAE) dans sa dernière édition du Dictionnaire de la Langue Espagnole.

\section{Abstract:}

In this paper we will present the collection of part of the lexicon related to the manufacturing process of the Peruvian wines in order to achieve a basic understanding of this topic. For data collection, it was considered necessary to develop a questionnaire whose application was carried out in some wineries located in the city of Lima. Once the necessary corpus was collected, it was systematized following different criteria stipulated by the Royal Spanish Academy (RAE) in the latest edition of the Dictionary of the Spanish Language.

Palabras clave: léxico; vinos; proceso; fabricación; vinos peruanos.

Mots clés: lexique; vins; processus; fabrication; vins péruviens.

Key words: lexicon; wines; process; manufacturing; Peruvian wines.

Fecha de recepción: $\quad$ 28/02/2013

Fecha de aceptación: $\quad$ 02/04/2013

\section{PREÁMBULO}

El presente trabajo constituye una recopilación del léxico relacionado con el proceso de fabricación de vinos.

A lo largo de los años se han realizado trabajos de esta índole con el objetivo de poder compilar, en un solo material, la mayoría de los vocablos que se encuentran insertados dentro de este campo. Sin embargo, la mayor parte de estos trabajos se ha remitido, simplemente, a hacer un recojo de entradas sobre los diversos tipos de vinos existentes, dejando de lado aspectos como los referidos a los constituyentes del vino, sus etapas de fabricación o los diferentes tipos de uva a partir de los cuales son elaborados. En este trabajo se incluirán una serie de entradas que se encuentran adscritas dentro de estos campos. 
Para llevar a cabo la presente empresa, se pasaron una serie de etapas que detallaremos a continuación:

\section{Primera etapa. Revisión bibliográfica}

Se realizó una revisión minuciosa de algunos de los trabajos elaborados sobre el tema en cuestión con la finalidad de tener un conocimiento de partida sobre todo aquello que guarda algún tipo de relación con el campo de la enología.

\section{Segunda etapa. Elaboración del cuestionario}

A partir de los datos encontrados durante la revisión bibliográfica, se elaboró un cuestionario cuya aplicación nos permitió obtener todas las entradas que se encuentran consignadas en el presente léxico.

\section{Tercera etapa. Aplicación del cuestionario}

Esta etapa se llevó a cabo en la Antigua Taberna Queirolo ubicada en la Av. San Martín 1090, Pueblo Libre, Lima, Perú. Las personas que colaboraron para la realización de la presente etapa fueron Jorge Felipe Queirolo Targarona y Luis Francisco Queirolo Ciuffardi quienes son enólogos de profesión y supervisan cada una de las etapas que conlleva el proceso de producción de vinos.

\section{Cuarta etapa. Construcción de la estructura final del trabajo}

La estructura final del trabajo comprende las siguientes partes:

\section{Preámbulo}

Abreviaturas y símbolos. Aquí se presenta un listado de las abreviaturas que serán utilizadas en la estructura de cada uno de los artículos consignados en el léxico. 
Breve historia de los vinos. Se presentará una breve remembranza sobre los vinos desde sus posibles orígenes hasta su situación actual.

Cuerpo del trabajo. Constituye el núcleo del trabajo. En él se encuentran consignados todos los artículos del presente léxico (los mismos que suman un total de 102 entradas). Para llevar a cabo el proceso de lematización se ha tomado en cuenta los criterios (solo los pertinentes para este trabajo) empleados por la Real Academia Española (RAE) para la elaboración de su diccionario (DRAE). Estos son los siguientes:

- Los artículos se encuentran ordenados semasiológicamente, es decir, siguiendo el orden alfabético tradicional.

- El lema que constituye la cabeza del artículo se encuentra escrito en letra negrita redonda. Solo cuando se trate de palabras provenientes de otras lenguas que no han sido adaptadas al castellano (extranjerismos crudos), el lema aparecerá escrito en letra negrita cursiva (en este caso se brindará la información etimológica encerrada siempre dentro de los paréntesis).

- En seguida, aparecen la acepción o acepciones correspondientes al lema en cuestión. Si un mismo lema presenta más de una acepción, cada una de estas serán separadas por una doble barra vertical y enumeradas a partir de la segunda acepción.

Ejemplo:

bodega. f. Lugar donde se guarda y se cría el vino. II 2. Establecimiento donde se elaboran los vinos.

- Se hará uso de la virgulilla $(\sim)$ como sustituto del lema solo en los lemas internos mas no cuando aquel aparece en el texto definitorio de las acepciones en cuyo caso aparecerán en letra negrita.

Ejemplo:

vino. $\mathrm{m}$. [...] II $\sim$ aromático. $\mathrm{m}$. vino que presenta gran riqueza de aromas. [...] 
Conclusiones. De forma general, se dará cuenta de los resultados principales a los que nos ha conducido esta investigación.

Anexos. Aquí se incluye la bibliografía, el cuestionario que se aplicó en el campo, un modelo de ficha léxica y un modelo de ficha sociolingüística.

Es importante señalar, finalmente, que el presente léxico está dirigido a toda persona que desee encontrar información relacionada con el proceso de fabricación de vinos y no solamente a los especialistas en esta materia.

\section{HISTORIA DEL VINO}

Una bebida tan antigua como la civilización misma, con más de veinticinco siglos de historia, en la que la religión — divinidades, monasterios, monjes y cultos - ha estado presente desde sus orígenes. El cultivo de la vid ba recorrido la faz de la Tierra, trasladándose de pueblo en pueblo.

El vino está ligado al hombre desde épocas muy remotas. En Asia Menor, en las regiones cercanas al mar Caspio y al mar Negro, aparecen vestigios de viñas de unos 12000 años a. C.

Establecer el origen del vino es una tarea muy difícil, pero sí puede rastrearse su historia a través de las diferentes civilizaciones. La elaboración del vino se fue abriendo paso en Europa con la expansión del Imperio romano a lo largo de todo el Mediterráneo, momento en el que se establecieron muchas de las principales regiones vinícolas que aún existen en la actualidad.

Ya en aquel entonces, la elaboración del vino era un oficio muy preciso que fue promocionando el desarrollo de nuevas variedades de uva y de las técnicas de cultivo. Fueron apareciendo así los toneles para la reserva y el transporte del vino, además de las botellas que se utilizaron en ese entonces por primera vez e incluso se creó un rudimentario sistema de denominación, según fueron adquiriendo reputación algunas regiones de 


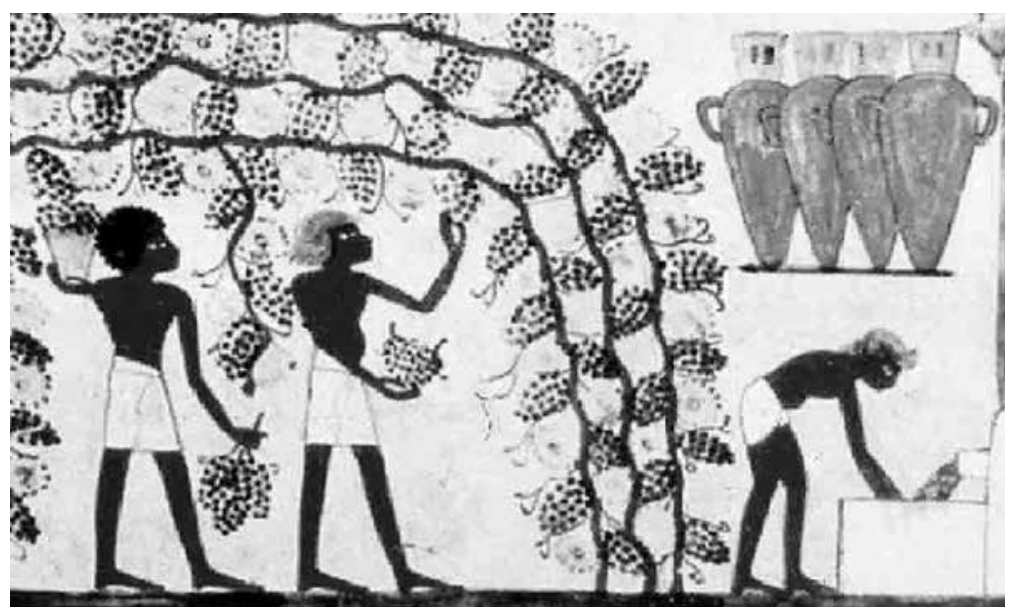

producción de vinos finos. Conforme se fue refinando progresivamente la producción de esta bebida, se incrementó su popularidad y las tabernas de vino pasaron a ser un elemento común de todas las ciudades del Imperio.

Ahora bien, la cultura del vino es anterior a la época de los romanos en Europa. Efectivamente, ya en la Grecia antigua, el vino era loado por los poetas, los historiadores y los artistas y aparece con frecuencia en las obras de Esopo y Homero. No obstante, en Grecia, el vino se consideraba como privilegio de las clases superiores. Dionisio, el dios griego del vino, representaba no solo los poderes de intoxicación de esta bebida, sino también sus influencias sociales y benéficas. Se le consideraba como el dios promotor de la civilización, la longevidad, el amor y la paz, al igual que el santo patrón de la agricultura y del teatro. De hecho, de acuerdo con el historiador griego Tucídides, "las gentes del Mediterráneo empezaron a emerger del barbarismo cuando aprendieron a cultivar el olivo y la vid".

Los relieves de Ur, el código de Hammurabi de la cultura mesopotámica y las pinturas y papiros de Egipto incluían el vino como un producto de uso cotidiano. Incluso en el Antiguo Testamento abundan las menciones al vino. Los griegos también contribuyeron a su evolución, ya que sus métodos eran mejores. El dios griego del vino, Dionisio, fue 
llamado Baco en Roma. Los romanos, que sometían sus vinos a curiosas y hasta poco atractivas o incluso peligrosas técnicas de conservación, apreciaron, sin embargo, los vinos de las Galias, Hispania y del resto de Europa y del norte de África. No solo exportaron los vinos, sino que también adoptaron las técnicas de fabricación.

Con el paso de los siglos, el arte de elaborar vino se fue extendiendo en Francia, España, Alemania y parte de Bretaña. En esa época, ya se consideraba a esta bebida como una parte importante de la dieta diaria y la gente empezó a apreciar los vinos más fuertes y de más carácter. La apreciación del vino en Europa se afianzó con la Edad Media, en parte porque beber agua todavía no era seguro, por lo que el vino era la alternativa preferida para acompañar las comidas. Al mismo tiempo, la viticultura y la vinicultura fueron avanzando gracias a la labor de los monasterios repartidos por el continente, donde nacieron algunos de los viñedos más finos de Europa. Por ejemplo, los monjes benedictinos fueron unos de los productores de vino más importantes de Europa, con viñedos en las regiones de Champagne, Borgoña y Burdeos, así como en las regiones alemanas del Rin y de Franconia. Las clases nobles y los comerciantes bebían vino en cada comida y tenían sus bodegas bien abastecidas.

En China conocían el vino y, de hecho, en el año 2285 a. C. ya habían establecido una reglamentación para evitar adulteraciones; se contemplaban serios castigos para quienes mezclaran vino de uva con vino de arroz.

Durante el siglo XVI empezó a apreciarse el vino como una alternativa más refinada que la cerveza. Según se fueron diversificando los productos de la vid, los consumidores empezaron a valorar la variación de sus hábitos de consumo, y la gente empezó a comentar los vicios y las virtudes del vino con mayor entusiasmo que en siglos pasados. El tan famoso vate de la Inglaterra de la época isabelina observaba ya que "el buen vino es una jovial criatura, si de él se hace buen uso”, comentando implícitamente sobre el uso indebido del vino en aquel entonces. Durante la época shakespeariana empezó a disponerse de agua potable en Londres, adelanto que trasladó el sector del vino a una nueva era. 
La mejora de las técnicas de producción intervenidas durante los siglos XVII y XVIII desembocaron en la elaboración de vinos más refinados; empezaron a utilizarse las botellas de vidrio y se inventaron los tapones de corcho. El auge del sector del vino francés se inició en este período, al darle los comerciantes de los Países Bajos, Alemania, Irlanda y Escandinavia un reconocimiento particular a los vinos de la región de Burdeos. Esta zona comerciaba con el vino a cambio de café y otros objetos codiciados procedentes del Nuevo Mundo; lo cual contribuyó a reforzar el papel del vino en el emergente comercio mundial.

Durante los ciento cincuenta últimos años, la elaboración del vino ha sido totalmente revolucionada como arte y ciencia que es. Efectivamente, con el acceso a la refrigeración, las bodegas han podido controlar fácilmente la temperatura y los procesos de fermentación y producir vinos de alta calidad en regiones de clima cálido. La introducción de maquinaria para la recolección ha propiciado la extensión y la mayor eficacia de las viñas. Si bien el sector del vino debe afrontar el reto de satisfacer la demanda de un creciente mercado sin perder el carácter individual de sus vinos, la tecnología contribuye a asegurar una oferta uniforme de vinos de calidad. La apreciación en que se tiene actualmente al vino es un tributo pagado al arte intemporal de su elaboración y demuestra la importancia de esta bebida en la historia y en la diversidad de la cultura europea.

\title{
LISTA DE ABREVIATURAS Y SIGNOS EMPLEADOS
}

\author{
f. femenino; nombre femenino \\ m. masculino; nombre masculino \\ tr. transitivo; verbo transitivo \\ Signo de palabra \\ II Separación de acepciones del mismo bloque \\ fr. francés \\ lat. latín; latino \\ Esp. España \\ Fórm. fórmula
}


A

abra. f. Espacio libre que queda entre las barricas.

acabado. m. Etapa final del proceso de elaboración de los vinos.

acidez. f. Cantidad de ácido contenida en el vino.

acidificación. f. Aumento de la acidez natural del mosto y de los vinos por procedimientos físicos, químicos o biológicos.

acodar. tr. Enterrar parte de un vástago sin separarlo de la planta de vid.

aguar. tr. Mezclar agua con vino.

alcornoque. m. Árbol de la familia fagáceas, de madera muy dura, hojas persistentes, fruto en bellota, y cuya corteza constituye el corcho.

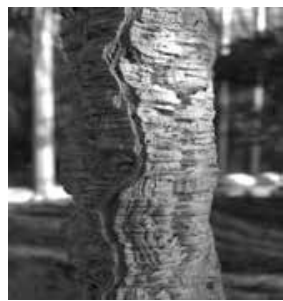

ampelografía. m. Ciencia que estudia las variedades de uva y su hoja.

ampelología. f. Ciencia que estudia la biología de la vid, su cultivo, el origen geográfico de las diferentes variedades de cepa, su adaptación a los suelos, sus patologías y tratamientos.

andana. f. Esp. Conjunto de barricas apiladas unas sobre otras hasta formar un máximo de cinco filas.

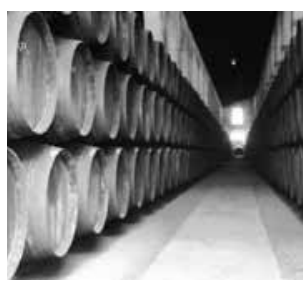


anhidrido carbónico. Gas que se forma durante la fermentación alcohólica de los mostos. (Fórm. $\mathrm{CO}_{2}$ ).

anillo. m. Moldura circular que refuerza el gollete de la botella de vino para que resista la presión que se ejerce al encorcharla.

antociano. m. Sustancia colorante que se encuentra en la piel de las uvas tintas, y es responsable del color de los vinos tintos.

asoleamiento. m. Fase del manejo de cultivo de la uva que consiste en la acumulación de azúcares en el fruto, a través de la interceptación de radiación solar realizada por el follaje.

azucarar. tr. Añadir azúcar a los mostos de baja calidad con el fin de obtener mayor cantidad de alcohol durante el proceso de fermentación.

barrica. f. Tonel de tamaño mediano en el que se añeja el vino.

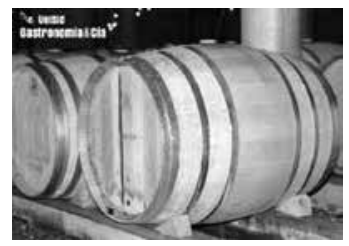

bocoy. (Del fr. boucaut, tonel). m. Esp. Recipiente cilíndrico de Madera,

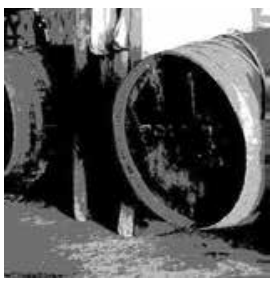
cuya capacidad sobrepasa los quinientos litros. Sirve para almacenar o transportar vinos.

bodega. f. Lugar donde se guarda y se cría el vino. II 2. Establecimiento donde se elaboran los vinos. 
bojo. m. Barriga o curvatura lateral de una barrica.

botella. f. Vasija de cuello angosto, hecha de cristal, vidrio u otro material, que sirve para contener el vino.

brazo. m. Rama de la cepa de la cual salen los sarmientos.

capa. f. Intensidad o profundidad del color de un vino.

cava. (Del lat. cava, zanja). f. Técnica de cultivo de la vid que consiste en cavar los alrededores de la cepa separando la tierra que cubre el tronco.

cepa. f. Tronco de la vid del cual brotan los sarmientos, las hojas y los frutos. II 2. Variedad de planta de viña. II madre. f. Cepa destinada a suministrar sarmientos para injertos.

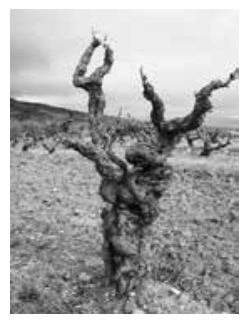

clarificar. tr. Filtrar los vinos para garantizar su perfecta limpidez.

concentración. f. Eliminación de la mayor parte de agua contenida en los mostos.

cono. m. Recipiente de hormigón, acero inoxidable o madera, cuya capacidad es aproximadamente entre ocho mil y veinte mil litros. Sirve para almacenar vinos.

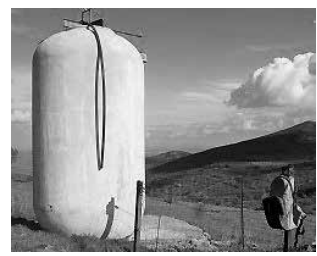

contraetiqueta. f. Sello acreditativo colocado en la parte posterior de la botella que certifica a aquellos vinos que han superado los niveles de calidad exigidos. 
corcho. m. Parte exterior de la corteza del alcornoque.

II 2. Tapón que se fabrica con esta corteza.

crianza. f. Proceso controlado de envejecimiento y maduración de un vino que tiene lugar en barricas, botas o botellas a través del cual se desarrollan caracteres especiales.

cromatografía. f. Método de análisis químico que se aplica a los vinos para fraccionar su composición con el fin de efectuarle controles de calidad.

\section{D}

decolorar. tr. Atenuar la intensidad del color de un vino por añadidura de ciertas sustancias.

desacidificación. f. Reducción de la acidez de un vino por procedimientos físicos, químicos o biológicos.

desecación. f. Deshidratación de las uvas.

despalillar. tr. Separar de los racimos de uva la parte leñosa antes de que sean estrujados.

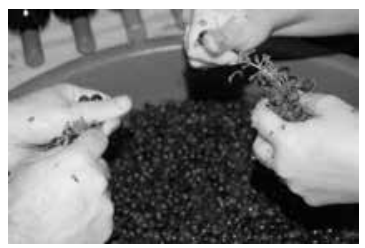

duela. f. Cada una de las tablas que forman la pared curva de las barricas.

E

embodegar. tr. Guardar el vino en la bodega.

embotellar. tr. Envasar el vino en botellas.

encorchar. tr. Colocar tapones de corcho a las botellas. 
envero. (De enverar). m. Color que toman las uvas al comenzar su proceso de maduración.

envinada. f. Barrica que posee una antigüedad de dos años o más.

envinar. tr. Echar vino en el agua. II 2. Llenar de vino las barricas o envases de crianza.

escurrir. tr. Dejar fluir, gota a gota, el mosto que se desprende de las uvas luego de la vendimia.

espaldera. f. Sistema de plantación en el que del tronco de la cepa salen dos ramas principales que se alinean en el sentido de una hendidura, atándose a alambres cercanos al suelo y conectados entre sí.

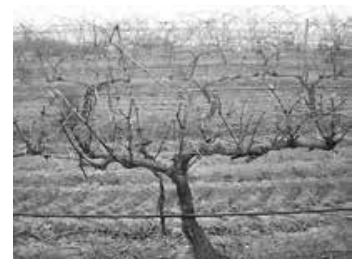

esperriaca. f. Mosto final que se extrae de la uva.

espita. f. Tubo estrecho y pequeño que se abre o cierra por el giro de

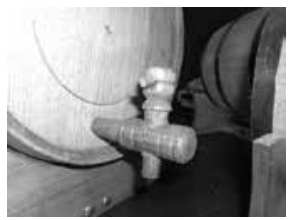
una llave o mediante una palanca y que se coloca en el agujero de un tonel, una barrica o un recipiente cualquiera para su posterior vaciado.

espuma. f. Capa que se forma en la superficie de los vinos durante su fermentación.

estrujar. tr. Triturar las uvas a fin de extraer el mosto.

\section{F}

fermentación. f. Transformación de los azúcares del mosto en alcohol. II maloláctica. f. Transformación del ácido málico en ácido láctico.

Bol. Acad. peru. leng. 55(55), 2013 
follaje. m. Conjunto de hojas de los árboles y otras plantas de vid.

\section{G}

gollete. m. Cuello estrecho que tienen las botellas de vino.

graduación. f. Proporción de alcohol contenida en un vino.

\section{$\mathbf{H}$}

hollejo. m. Piel que envuelve la pulpa del grano de uva y que, además, contiene pigmentos, aromas y taninos.

\section{I}

ictiocola. f. Cola de pescado procesada químicamente en una solución gelatinosa. Contribuye al clarificado de los vinos.

impurezas. f. Materias extrañas que pueden aparecer en un vino por accidente.

$\mathbf{L}$

lagar. f. Sitio donde se realizan la molienda y el estrujado de la uva. II 2. Recipiente destinado para pisar las uvas y así obtener el mosto. II 3. Espacio en una bodega en donde se ubican las prensas.

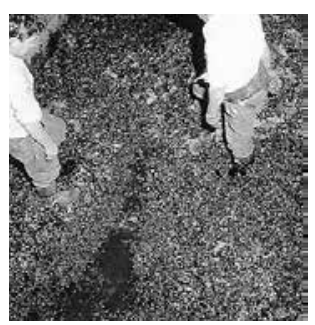

levadura. f. Masa constituida por microorganismos del grupo de hongos unicelulares, capaces de desencadenar la fermentación alcohólica en los hollejos de las uvas. II 2. Aroma de un fermento.

lía. f. Resto de levadura, ya sin actividad, que se deposita en el fondo de las barricas luego de producida la fermentación. II 2. Sabor de los vinos que han permanecido mucho tiempo en contacto con los sedimentos. 
lloro. $\mathrm{m}$. Segregación de savia por parte de la cepa a causa de las heridas de la poda, la cual se realiza antes del brote de alguna variedad de cepa.

\section{M}

maceración. f. Inmersión más o menos prolongada de los hollejos en el mosto cuyo fin es lograr una mayor extracción de todos los componentes que se encuentran en la piel de la uva. II $\sim$ fermentativa. f. Maceración que realizan todos los vinos tintos durante la fermentación alcohólica. II no fermentativa. f. Maceración que realizan tanto los vinos tintos como los vinos blancos ya sea antes o después de la fermentación alcohólica.

maduración. m. Período del proceso de desarrollo de la uva que comprende desde el envero hasta el estado de madurez fisiológica o parada de migración de azúcares hacia el grano.

mecha. f. Aparato que contiene azufre y se utiliza para desinfectar las barricas.

mejer. tr. Remover las lías para que estén en contacto permanente con el mosto.

melga. $f$. Sector de viña que se le confiere a un viticultor para que realice las labores de poda, regadío, cosecha, etc.

merma. f. Término empleado para designar a una vasija, barrica o botella cuya capacidad no está totalmente colmada.

mosto. m. Jugo o zumo de uva que no ha sufrido fermentación alguna.

$\mathrm{O}$

orujo. m. Conjunto de residuos sólidos obtenidos luego del prensado de los granos de uva. 
oxidar. tr. Alterar el aroma y el color de los vinos a través del excesivo contacto de estos con el aire.

\section{$\mathbf{P}$}

pabellón. m. Sistema de palos, estacas y alambres que permiten enredar los sarmientos de la cepa evitando así el arrastre de los racimos por el suelo.

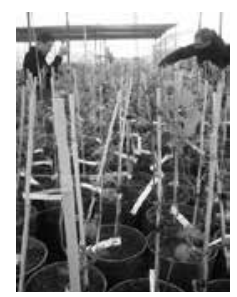

parra. f. Conjunto de tallos que trepan a lo largo de muros y vallas por medio de órganos especializados llamados zarcillos.

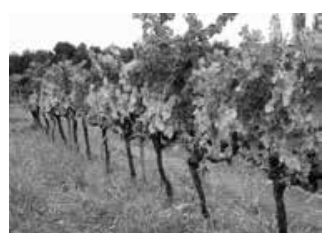

pedúnculo. $\mathrm{m}$. Soporte del grano de uva formado por taninos que le sirven de alimento.

pepita. f. Parte del grano de uva cubierta de pulpa que difiere en volumen y cantidad según las variedades de uva existentes.

podar. tr. Eliminar partes de la vid para conseguir un mayor rendimiento de la planta.

prensa. f. Máquina empleada para extraer el mosto de las uvas mediante presión.

prensar. tr. Extraer el mosto de las uvas mediante presión.

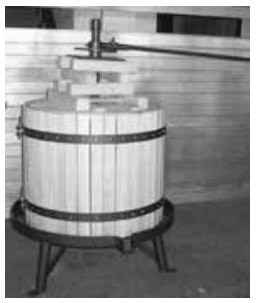

pulpa. f. Parte carnosa y jugosa de los granos de uva. 


\section{$\mathbf{R}$}

racimo. m. Conjunto de granos de uva sostenidos por un mismo pedúnculo.

raspón. $\mathrm{m}$. Parte leñosa del racimo que forma su estructura o esqueleto.

refrigeración. m. Proceso enotécnico utilizado para evitar temperaturas excesivas durante la fermentación de los mostos.

rimero. $\mathrm{m}$. Bloque compacto de botellas apiladas en forma horizontal.

\section{S}

savia. f. Líquido espeso que circula por los vasos conductores de las plantas superiores cuya función es nutrirlas.

sobremaduración. m. Período en el cual la uva permanece un tiempo desmedido en la cepa.

\section{$\mathrm{T}$}

tanino. $\mathrm{m}$. Sustancia astringente, de naturaleza vegetal, contenida en los hollejos, raspones y pepitas de la uva.

tolva. f. Recipiente generalmente metálico y con forma de pirámide truncada que sirve para depositar las uvas recién vendimiadas.

tonelero. $\mathrm{m}$. Persona que construye las barricas de

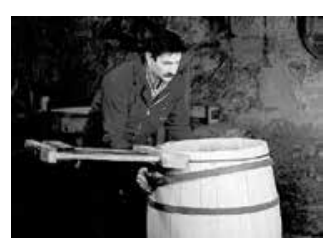
roble que se utilizan para el añejamiento del vino.

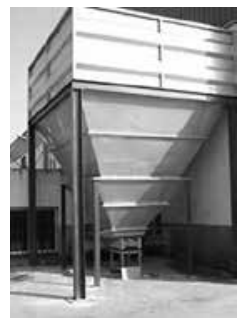


trasegar. tr. Pasar el vino de una vasija a otra para clarificarlo.

\section{$\mathrm{U}$}

uva. f. Fruto de la vid utilizado en la elaboración de vinos.

uvero. m. Esp. Sarmiento largo que resulta de la poda de cepas cuya función es producir más racimos.

\section{V}

vástago. m. Rama tierna de un árbol o planta. II 2. Conjunto de tallo y hojas.

vendimia. f. Recolección de uvas en las viñas.

vid. f. Arbusto constituido por raíces, tronco, sarmientos, hojas, flores y fruto o uva.

vidueño. $m$. Sector del viñedo cuyas plantaciones de cepa pertenecen a una misma variedad.

vinicultura. f. Ciencia que se encarga de estudiar el proceso de elaboración del vino.

vinificación. f. Transformación del mosto de la uva en vino.

vino. m. Bebida alcohólica que se obtiene a partir del zumo de uva exprimido y fermentado. II aromático. m. Vino que presenta gran riqueza de aromas. II $\sim$ complejo. m. Vino que posee diferentes matices en su aroma. II $\sim$ cosechero. m. Vino con un máximo de seis meses de añejamiento en barrica de roble. II de guarda. $\mathrm{m}$. Vino con un año de añejamiento en barrica de roble. II efervescente. $\mathrm{m}$. Vino con presencia de burbujas. II equilibrado. m. Vino que presenta armonía entre todos sus componentes. II $\sim$ especiado. $\mathrm{m}$. Vino que posee aromas y sabores de especies. II $\sim$ gran reserva. m. Vino con más de dos 
años de añejamiento en barrica de roble y tres años más en botella. II licoroso. m. Vino que presenta en su composición más de cincuenta gramos de azúcar por litro de vino. II ligero. $\mathrm{m}$. Vino que posee poco alcohol en su composición. II $\sim$ meloso. m. Vino que presenta en su composición entre treinta y cincuenta gramos de azúcar por litro de vino. II $\sim$ mezclado. m. Vino que ha sido elaborado con al menos dos variedades de uva. II pasado. $\mathrm{m}$. Vino que ha sufrido alteraciones y se encuentra en malas condiciones. $I I \sim$ reserva. $\mathrm{m}$. Vino con un año de añejamiento en barrica de roble y dos años más en botella. II seco. $\mathrm{m}$. Vino que presenta en su composición menos de dos gramos de azúcar por litro de vino. II semiseco. m. Vino que presenta en su composición entre dos y treinta gramos de azúcar por litro de vino. II $\sim$ tranquilo. $\mathrm{m}$. Vino sin presencia de burbujas. II $\sim$ varietal. $\mathrm{m}$. Vino elaborado con un solo tipo de uva.

vinómetro. $\mathrm{m}$. Instrumento utilizado para medir la graduación alcohólica de los vinos.

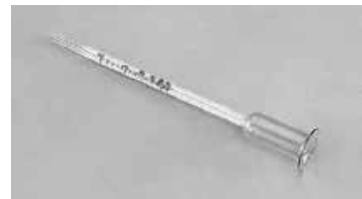

viña. f. Zona de plantación de vides para la producción de uvas.

virote. $\mathrm{m}$. Esp. Tallo nuevo de la vid.

viticultura. f. Ciencia que se consagra al estudio y conocimiento de la planta de la vid y de las labores necesarias para que su crecimiento y fructificación sean satisfactorios.

vitivinicultura. f. Estudio del cultivo de la uva, además de la fabricación y cata de vinos.

\section{Y}

yema. f. Fracción inicial del mosto, obtenida mediante escurrido, sin que la pasta sufra presión alguna. II 2. Brote del sarmiento de la vid sin desarrollarse. 


\section{Z}

zarcillo. m. Ramificación filiforme delgada de la vid con tendencia a

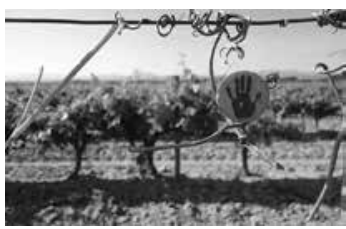
enrollarse en otras ramas a fin de buscar un soporte en donde sostenerse.

\section{CONCLUSIONES}

1. El proceso de producción de los vinos comprende una serie de campos que se constituyen en grandes fuentes de riqueza léxica, a saber: los tipos de cepa y de uva empleadas en su fabricación, los diferentes tipos de vino que se pueden elaborar a partir de aquellas y las diferentes etapas que conlleva este proceso (dentro de las cuales se hace uso de instrumentos y lugares específicos).

2. La mayoría del léxico relacionado con el proceso de elaboración de los vinos es de carácter patrimonial. Son muy pocos los casos en los que se hace uso de palabras provenientes de lenguas extranjeras.

3. El mayor grupo de lemas presentes en este recojo léxico tiene una relación de uno a uno con las definiciones; es decir, a cada lema le corresponde tan solo un significado. Escasos son los lemas que presentan dos o más acepciones. 


\section{BIBLIOGRAFÍA}

HAENSCH, Gunther et al. La Lexicografía. De la lingüística teórica a la lexicografía práctica. Madrid, Gredos, 1982.

MARTÍNEZ DE SOUZA, José. Diccionario de lexicografía práctica. Barcelona, Real Academia Española, 1995.

. Diccionario de la lengua española. Madrid, Espasa Calpe S. A., 2003.

ROJAS DOMÍNGUEZ, Leonor. Estudio del léxico de la hípica. Tesis para obtener la licenciatura en Lingüística. Lima, Universidad Nacional Mayor de San Marcos, 1996.

\section{Correspondencia:}

\section{Zamia Castillo Farroñay}

Bachiller en Lingüista por la Facultad de Letras y Ciencias Humanas de la Universidad Nacional Mayor de San Marcos.

Correo electrónico: as-samy_2008@hotmail.com 